\title{
Evaluación de la calidad del agua de la laguna Marvilla en los Pantanos de Villa (Lima, Perú)
}

Evaluation of the water quality of Laguna Marvilla in Pantanos de Villa (Lima - Peru)
Shanndy Huaman-Vilca ${ }^{1,4}{ }^{\oplus}$, Musye Lucen-Espinoza ${ }^{1,4 *}$, Mariela Paredes-Vite ${ }^{2,4}$ y Daniela Changanaqui Alfaro ${ }^{3}{ }^{\prime}$
1 Departamento de Ciencias de la Salud y la Vida, Universidad Científica del Sur. Lima, Perú.

2 Facultad de Ingeniería Agraria, Universidad Católica Sedes Sapientiae. Lima, Perú.

3 Autoridad Municipal Los Pantanos de Villa. Lima, Perú.

\section{SOUTH} SUSTAINABILITY

Citar como: Huaman-Vilca, S. et al. (2020). «Evaluación de la calidad del agua de la laguna Marvilla en los Pantanos de Villa (Lima, Perú)». South Sustainability, 1(2), e019 DOI: $10.21142 /$ SS-0102-2020-019

Artículo recibido: $27 / 11 / 2020$ Revisado por pares Artículo aprobado: 25/1/2021

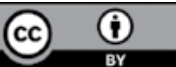

(c) Los autores, 2020. Publicado por la Universidad Científica del Sur (Lima, Perú)

\section{${ }^{*} E$-mail de correspondencia:} musye0998@gmail.com

\section{RESUMEN}

El Refugio de Vida Silvestre Los Pantanos de Villa es un humedal reconocido por albergar una significativa diversidad biológica y brindar importantes servicios ecosistémicos. Sin embargo, el crecimiento urbano y las actividades productivas que se desarrollan en sus alrededores generan un impacto en sus diferentes componentes, entre ellos la calidad del agua. Por ello, el objetivo de la investigación es evaluar la calidad del agua de la laguna Marvilla, a través de la comparación con los estándares de calidad ambiental (ECA) para agua y la determinación del índice de calidad de agua del Perú (ICA-PE) propuestos por la Autoridad Nacional del Agua (ANA). Se analizó la data anual de 2019-2020 y se obtuvieron promedios en los parámetros de $\mathrm{pH}$, conductividad eléctrica y sólidos disueltos totales de 8,8,4644,8 $\mu \mathrm{S} / \mathrm{cm}$ y 3413,9ppm, respectivamente. Además, se realizó un muestreo puntual de nueve parámetros fisicoquímicos y microbiológicos, de los cuales cinco no cumplen con lo establecido en la categoría 4 del ECA para agua (nitratos, fósforo total, amoniaco total, demanda bioquímica de oxígeno y coliformes termotolerantes). Asimismo, con los datos del muestreo se obtuvo un valor de 46,3 en el ICA-PE. Se concluye que la calidad de agua de la laguna Marvilla es regular, es decir, se encuentra ocasionalmente amenazada o dañada.

Palabras clave: humedal, agua, calidad, estándares, índice, parámetros

\section{ABSTRACT}

The Pantanos de Villa Wildlife Refuge is a wetland recognized for its significant biological diversity and important ecosystem services. However, urban growth and the industrial activities that are developed in the areas surrounding this wetland generate an impact upon its different components, including water quality. Thus, the aim of this research is to evaluate the water quality of Laguna Marvilla, through comparison with environmental quality standards (ECA) for water and the determination of the Peruvian Water Quality Index (ICA-PE) proposed by the National Water Authority (ANA). The annual data for 2019-2020 was analyzed in order to obtain averages for $\mathrm{pH}$ parameters, electrical conductivity and total dissolved solids: $8.8,4644.8 \mu \mathrm{S} / \mathrm{cm}$ and $3413.8 \mathrm{ppm}$ respectively. In addition, a punctual sampling was conducted of nine physicochemical and microbiological parameters, five of which did not meet the standards for water specified in Category 4 of the ECA, in terms of nitrates, total phosphorus, total ammonia, biochemical oxygen demand, and thermotolerant coliforms. Using the sampling data, an ICA-PE value of 46.35 was obtained, leading to the conclusion that the water quality of Laguna Marvilla is fair, and that it is punctually threatened or damaged.

Keywords: wetland, water, quality, standards, index, parameters 


\section{Introducción}

Los humedales costeros se originan a consecuencia del surgimiento de las aguas de la capa freática en zonas muy cercanas al mar (Arana y Salinas, 2003). Sin embargo, a pesar de que tienen un papel fundamental en el control de la erosión costera, el transporte de sedimentos, la regulación de inundaciones, la purificación de agua y el efecto que ejerce en el cambio climático (Russi et al., 2013), se calcula que el $50 \%$ de los humedales a nivel mundial se están perdiendo (Finlayson y D'Cruz, 2005) a causa de las acciones antrópicas, debido a que en la zona costera se asienta la mayor cantidad de ciudades del país (Arana y Salinas, 2003). Asimismo, investigaciones realizadas en tres humedales ubicados en Valdivia, Chile, refieren que los principales factores que inciden en su afectación son los continuos depósitos de basura, las construcciones, el incremento de especies vegetales exóticas y la presencia de animales domésticos (caballos y ganado) que se sitúan alrededor (Paredes, 2010).

El Refugio de Vida Silvestre Los Pantanos de Villa es un humedal ubicado en la costa peruana al sur de la zona urbana de Lima. Es un sitio Ramsar reconocido por albergar una importante y significativa diversidad biológica. Brinda servicios ecosistémicos como la captura de carbono, filtración y purificación de agua, y también contribuye a la mitigación de inundaciones y erosión costera, los cuales generan una mejor calidad de vida para las personas (Sajurjo, 2001). El factor hídrico es imprescindible para la vida de la flora y fauna del Refugio de Vida Silvestre Los Pantanos de Villa, puesto que la calidad de sus aguas, en conjunto con sus características fisicoquímicas y microbiológicas, influyen de forma directa en el correcto desarrollo de la biodiversidad (Pulido y Bermúdez, 2018). Por otro lado, el constante crecimiento urbano en la zona de amortiguamiento, que en su mayoría se trata de urbanizaciones, fábricas y camales que rodean el área natural protegida, además de la falta de conciencia y conocimiento en relación con la conservación del humedal, traen como consecuencia la merma en las características del ecosistema, principalmente la contaminación de los recursos hídricos (Ingemmet, 2019). Por tal motivo, nace la necesidad de evaluar la calidad de las aguas del humedal, con el fin de conocer la capacidad que tiene este recurso para ser utilizado en función de sus características físicas, químicas y microbiológicas. El agua es evaluada a partir de datos obtenidos de oxígeno disuelto, coliformes termotolerantes, $\mathrm{pH}$, entre otros, y así se la compara con los estándares o normativas ambientales que nos permitan determinar si está dentro de las condiciones adecuadas para cierto uso (Cordy, 2001). En ese sentido, es importante hacer uso del índice de calidad ambiental (ICA), ya que es una herramienta que permite sintetizar una variedad de parámetros fisicoquímicos y microbiológicos en una expresión matemática, con el objetivo de determinar el estado de la calidad de agua del humedal (Pérez y Álvarez, 2018). Entre los ICA más utilizados se encuentra el planteado por el Canadian Council of Ministers of the Environment (CCME_WQI), que propone la determinación de la calidad de agua en cierto rango de tiempo, utilizando como factores el número de parámetros que superan el estándar que toman como referencia, el número de datos que no cumplen con el mencionado estándary la magnitud de superación (CCME, 2001). Como resultado se puede conocer si dicho cuerpo de agua se ubica en rangos de calidad: excelente (valor $=90-100)$, buena (valor $=75-89$ ), regular (valor $=45-74)$, mala (valor $=30-49)$ y pésima (valor = 0-29), para así generar planes en favor de su conservación (ANA, 2018).

Comprender el estado actual del recurso hídrico del humedal Pantanos de Villa es de suma importancia. Por ese motivo, la presente investigación tiene como objetivo evaluar la calidad del agua de una laguna del humedal, a través de la comparación con los estándares de calidad ambiental (ECA) para agua y la determinación del índice de calidad de agua del Perú (ICA-PE) propuesto por la ANA. Este estudio tendrá un alcance para la laguna Marvilla, hábitat caracterizado por poseer la mayor biodiversidad de aves de todo el humedal Pantanos de Villa, con el fin de proporcionar información, facilitar la toma de decisiones a las autoridades competentes y así aportar en la mejora de la gestión de la conservación del humedal.

\section{Materiales y métodos}

\section{Área de estudio}

El área de estudio es la laguna Marvilla (figura 1), que abarca una extensión de cuatro hectáreas de las 263,27 que posee el humedal Pantanos de Villa (Pulido y Bermúdez, 2018). Se tienen tres puntos de muestreo, tal como se muestra en la figura 1.

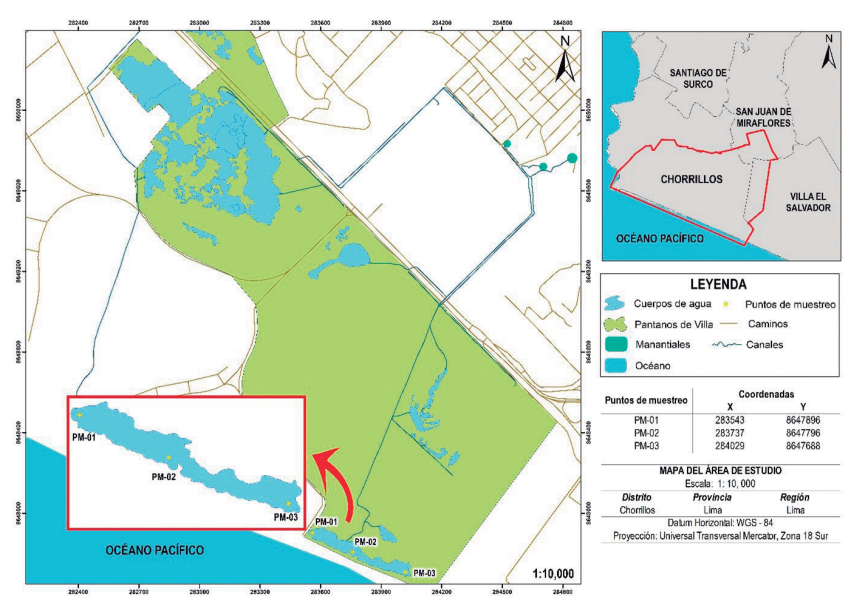

Figura 1. Mapa del área de estudio, laguna Marvilla, en el humedal Pantanos de Villa. 


\section{Métodos}

\section{Análisis 2019-2020}

La Autoridad Municipal de los Pantanos de Villa (Prohvilla) realiza monitoreos mensuales de la calidad de agua desde octubre de 2019. Los parámetros que evalúa en los tres puntos de muestreo ubicados en la laguna Marvilla (figura 1) son la conductividad eléctrica, los sólidos disueltos totales y el $\mathrm{pH}$.

Con el fin de analizar la data anual, se utilizaron los datos registrados mensualmente de conductividad eléctrica y $\mathrm{pH}$ de octubre a diciembre de 2019 y de enero a agosto de 2020, a excepción de los meses de marzo y abril, debido a que no se realizaron los monitoreos respectivos por las restricciones del Gobierno ante la pandemia de la covid-19. Estos datos fueron comparados con los valores establecidos en la categoría 4 («Conservación del ambiente acuático»), subcategoría E1 («Lagunas y lagos») del Decreto Supremo 004-2017-MINAM, «Estándar de calidad ambiental para agua» (Ministerio del Ambiente, 2017).

También se realizó la evaluación de la fluctuación del nivel del agua en la laguna Marvilla con base en los datos de monitoreos de la escala limnimétrica que realiza Prohvilla mensualmente. Se buscó identificar la temporada donde los niveles de agua en el humedal son bajos y altos en el periodo 2019-2020.

Para la determinación de los sólidos disueltos totales, Groel (2006) menciona que a través del valor de la conductividad eléctrica se puede obtener un aproximado de la cantidad de ppm presentes en el medio. Por ello, se procedió a calcular su valor de acuerdo con la siguiente fórmula dada por Wate Reuse (2007):

$$
\text { SDT }(\mathrm{mg} / \mathrm{L})=\mathrm{CE}(\mathrm{dS} / \mathrm{m}) \times 0,735
$$

Sin embargo, los valores de sólidos disueltos totales no fueron comparados con el ECA para agua, ya que este parámetro no se ha establecido en la categoría 4.

\section{Evaluación de los parámetros fisicoquímicos y microbiológicos}

Selección de los puntos de muestreo

Se consideraron los tres puntos de muestreo utilizados por Prohvilla en sus monitoreos mensuales de la calidad de agua en la laguna Marvilla (figura 1).

\section{Muestreo}

El muestreo se realizó en octubre de 2020 y de acuerdo con el Protocolo Nacional para el Monitoreo de la Calidad de los Recursos Hídricos Superficiales de la ANA (2016). Antes de iniciar con el procedimiento para la toma de muestras, se realizó el reconocimiento del entorno, la rotulación y el etiquetado de los frascos. Primero se midieron los parámetros de campo $(\mathrm{pH}$, temperatura, oxígeno disuelto, conductividad eléctrica y sólidos disueltos totales) in situ. Luego, se obtuvieron muestras en los recipientes para la medición de nitratos, fósforo totaly amoniaco total. Posteriormente, se procedió a recolectar las muestras en los frascos para la determinación de demanda bioquímica de oxígeno $\left(\mathrm{DBO}_{5}\right)$ y coliformes termotolerantes, cumpliendo con las especificaciones solicitadas (recolección, acondicionamiento y conservación) por el laboratorio R-LAB SAC.

Medición y determinación de los parámetros fisicoquímicos y microbiológicos

Para la medición in situ de $\mathrm{pH}$, temperatura $\left(\mathrm{T}^{\circ}\right)$, conductividad eléctrica (CE) y sólidos disueltos totales (SDT), se utilizó un multiparámetro Hanna HI 9813-6; para oxígeno disuelto (OD), un oxímetro Hach Pocket Pro; para nitratos, un test kit de prueba de nitrato Hach NI-11; para fósforo total, un test kit de prueba de ortofosfato PO19; y para amoniaco, un test kit de prueba de nitrógeno amoniacal Hach NI-SA. A su vez, para la determinación de la $\mathrm{DBO}_{5}$ y coliformes termotolerantes, se obtuvieron tres muestras por parámetro que fueron llevadas al laboratorio para su análisis. Las metodologías utilizadas por el laboratorio acreditado de R-LAB SAC, fueron las siguientes: $\mathrm{DBO}_{5}$ (SMEWW-APHA-AWWA-WEF Part 5210 $B$, 23rd Ed) y coliformes termotolerantes (SMEWW-APHAAWWA-WEF Part 9221 E-1, 23rd Ed).

Del mismo modo, los valores obtenidos fueron comparados con los valores establecidos en la categoría 4 («Conservación del ambiente acuático»), subcategoría E1 («Lagunas y lagos») del ECA para agua, a excepción de la temperatura; ya que, según la normativa, la determinación del parámetro debe contar con data de un año como mínimo. Asimismo, los sólidos disueltos totales tampoco fueron comparados con el ECA para agua, puesto que este parámetro no se ha establecido en la categoría 4.

\section{Determinación del índice de calidad de agua (ICA-PE)}

Se utilizó la metodología para la determinación del índice de agua ICA-PE aplicado a los cuerpos de agua continentales superficiales con respecto a un solo muestreo de la ANA (2018), la cual indica que para la aplicación del índice se debe utilizar un mínimo de cuatro parámetros. En ese sentido, los parámetros considerados para la aplicación del ICA-PE en la categoría 4, «Conservación del ambiente acuático», fueron $\mathrm{DBO}_{5}$, fósforo total, amoniaco total, $\mathrm{OD}, \mathrm{pH}$ y coliformes termotolerantes.

Para ello, se aplicó la siguiente fórmula para un monitoreo:

$$
I C A-P E=\frac{100-\sqrt{F_{1}^{2}+F_{2}^{2}+F_{3}^{2}}}{3}
$$


Donde:

$$
\mathrm{F}_{1}=\mathrm{F}_{2}
$$

Alcance $\left(F_{1}\right)=\frac{\text { Número de parámetros que no cumplen los ECA-Agua }}{\text { Número total de parámetros a evaluar }}$ Frecuencia $\left(F_{2}\right)=\frac{\text { Número de parámetros que no cumplen los ECA-Agua }}{\text { Número total de parámetros a evaluar }}$ Amplitud $\left(F_{3}\right)=\frac{\text { Suma normalizada de excedentes }}{\text { Suma normalizada de excedentes }+1} * 100$

Alcance y frecuencia son la misma fórmula para un solo muestreo. Por otro lado, la clasificación del índice de calidad de agua se presenta en la tabla 1.

\section{Resultados y discusión}

\section{Hidroperiodo de la laguna Marvilla}

Los resultados del hidroperiodo 2019-2020 (tabla 2) nos permiten observar que de junio a noviembre el nivel de agua tiende a incrementar. De diciembre a mayo el nivel de agua disminuye.

\section{Análisis de parámetros anuales}

Los valores de $\mathrm{pH}$ se sitúan entre un rango neutro y ligeramente alcalino. Según los resultados, en noviembre, diciembre, enero y mayo los valores sobrepasaron lo establecido en la categoría 4 del ECA para agua, el cual establece un rango entre 6,5 y 9 (figura 2).

En ese sentido, se evidencia que los valores de $\mathrm{pH}$ disminuyeron en los meses donde el nivel de agua en la laguna es alto (junio-noviembre), mientras que en los meses donde el nivel de agua es bajo (diciembre-mayo) los valores incrementaron. Asimismo, se muestra que la laguna Marvilla presenta una condición alcalina, debido a la cercanía del humedal con el mar. Pérez et al. (2017) lo confirman en su estudio, que evalúa la calidad de agua de un humedal ubicado en el Caribe. El trabajo afirma que el humedal presenta un agua alcalina, debido a que es abastecido por agua proveniente del mar Caribe. Por otro lado, los valores de conductividad eléctrica (tabla 3) sobrepasaron lo establecido en la categoría 4 del ECA para agua, ya que exceden el valor máximo de $1000 \mu \mathrm{S} / \mathrm{cm}$. Estos resultados coinciden con lo propuesto por

Tabla 1. Interpretación de la calificación ICA-PE.

\begin{tabular}{cc}
\hline ICA-PE & Calificación \\
\hline $90-100$ & Excelente \\
\hline $75-89$ & Bueno \\
\hline $45-74$ & Regular \\
\hline $30-44$ & Malo \\
\hline $0-29$ & Pésimo
\end{tabular}

Fuente: ANA (2018).
Ingemmet (2019), el cual menciona que valores altos en conductividad eléctrica están vinculados directamente con una alta salinidad presente en el suelo y en el aire de la laguna Marvilla. Otro factor determinante en el aumento de este parámetro es la cercanía que tiene al mar y la cantidad de sales disueltas, como calcio, sodio, entre otros factores. En cuanto a los sólidos disueltos totales, los valores obtenidos a través de la fórmula propuesta por Water Reuse (2007) estuvieron en un rango entre 3300 y 3550 ppm (tabla 3). De acuerdo con ello, se identificó que el agua de la laguna Marvilla, al estar entre el rango de 1000 y 5000 ppm, es salobre (tabla 4); es decir, agua con menor cantidad de sales que el agua de mar, pero con mayor cantidad de sales que el agua dulce (laguas, 2018). Esta característica es similar a la de lagunas Mejía del humedal costero de Arequipa y la laguna El Paraíso en Huacho (Cruz et al., 2007 y Sernanp, 2013).

Los valores obtenidos en la laguna Marvilla son similares o más elevados a los obtenidos por otros autores al realizar investigaciones en humedales costeros (tabla 5). El pH en los humedales ubicados en Trujillo es ligeramente menor a comparación de los humedales

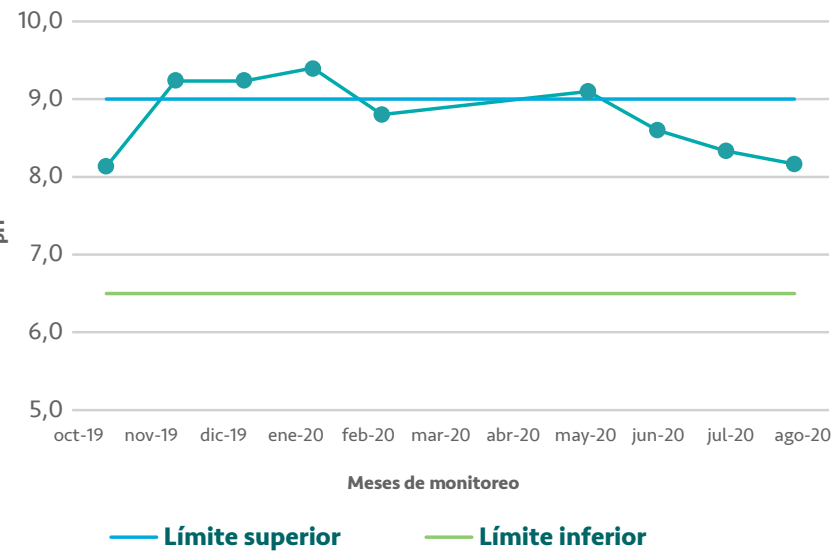

Figura 2. Variación del pH en la laguna Marvilla, Pantanos de Villa.

Tabla 2. Altura de agua en la laguna Marvilla.

\begin{tabular}{cc}
\hline Meses & Altura (cm) \\
\hline Octubre de 2019 & 48,5 \\
\hline Noviembre de 2019 & 49,0 \\
\hline Diciembre de 2019 & 23,0 \\
\hline Enero de 2020 & 33,0 \\
\hline Febrero de 2020 & 35,0 \\
\hline Abril de 2020 & 27,5 \\
\hline Mayo de 2020 & 38,0 \\
\hline Junio de 2020 & 49,5 \\
\hline Julio de 2020 & 51,5 \\
\hline Agosto de 2020 & 53,0
\end{tabular}

Fuente: Prohvilla. 
Tabla 3. Resultado de la medición de los parámetros de conductividad eléctrica y sólidos disueltos totales en el periodo 2019-2020.

\begin{tabular}{ccc}
\hline Meses/Parámetros & C. E. & SDT \\
\hline Octubre de 2019 & 4710,0 & 3461,9 \\
\hline Noviembre de 2019 & 4576,7 & 3363,9 \\
\hline Diciembre de 2019 & 4666,7 & 3430,0 \\
\hline Enero de 2020 & 4590,0 & 3373,6 \\
\hline Febrero de 2020 & 4546,7 & 3341,8 \\
\hline Mayo de 2020 & 4493,3 & 3302,6 \\
\hline Junio de 2020 & 4650,0 & 3417,7 \\
\hline Julio de 2020 & 4776,7 & 3510,9 \\
\hline Agosto de 2020 & 4793,3 & 3523,1 \\
\hline
\end{tabular}

Tabla 4. Clasificación de agua por nivel de salinidad.

\begin{tabular}{cc}
\hline Tipo de agua & Rango SDT (mg l-1) \\
\hline Agua dulce & $>550$ \\
\hline Agua salobre tolerable & $550-1000$ \\
\hline Agua salobre & $1000-5000$ \\
\hline Agua salina & $5000-35000$ \\
\hline
\end{tabular}

Fuente: Comisión Nacional del Agua. (2000); Water Quality Association (2010) citado por Álvarez (2015).

Tabla 5. Valores de parámetros de humedales costeros del Perú.

\begin{tabular}{ccccc}
\hline $\begin{array}{c}\text { Humedales } \\
\text { costeros del Perú }\end{array}$ & Autor & pH & C. E. & TDS \\
\hline $\begin{array}{c}\text { Campo Nuevo } \\
\text { (norte) }\end{array}$ & Bopp y Peláez (2019) & 7,5 & 535,0 & 330,0 \\
\hline $\begin{array}{c}\text { Balsares de } \\
\text { Huanchaco } \\
\text { (norte) }\end{array}$ & Bopp y Peláez (2019) & 7,7 & 702,0 & 603,5 \\
\hline $\begin{array}{c}\text { Pantanos de Villa } \\
\text { (centro) }\end{array}$ & Presente estudio & 8,8 & 4644,8 & 3413,8 \\
\hline $\begin{array}{c}\text { ACR Ventanilla } \\
\text { (centro) }\end{array}$ & Fajardo (2018) & 8,0 & 22045,9 & 19148,7 \\
\hline $\begin{array}{c}\text { Santa Rosa } \\
\text { (centro) }\end{array}$ & Huamantinco (2020) & 8,2 & 4219,1 & 2165,2 \\
\hline & & & & \\
\hline
\end{tabular}

costeros del centro del Perú. Sin embargo, tanto para el parámetro de conductividad eléctrica y sólidos disueltos totales, se observa mayor diferencia entre los humedales del norte con los del centro del Perú. Cabe mencionar que el ACR de Ventanilla mostró mayor concentración de CE y SDT de todos los humedales considerados.

\section{Evaluación de los parámetros fisicoquímicos y microbiológicos}

Los resultados de octubre de 2020 muestran que los parámetros de conductividad eléctrica, nitratos, fósforo total y coliformes termotolerantes sobrepasan el valor establecido en la categoría 4 del ECA para agua en los tres puntos de muestreo, así como el valor de $\mathrm{pH}$ en el PM-01 y el valor de amoniaco total, en el PM-02. Los valores obtenidos para cada uno de los parámetros se muestran en la tabla 6.

\section{Oxígeno disuelto}

Conforme con los resultados, los valores de oxígeno disuelto cumplen con la normativa, ya que superan el valor mínimo establecido de $\geq 5 \mathrm{mg} / \mathrm{L}$ en la categoría 4 del ECA para agua. Este parámetro es de suma importancia, puesto que cuando la concentración de oxígeno disuelto baja de $4 \mathrm{mg} / \mathrm{L}$, el agua no es apta para desarrollar vida (Jiménez, 2000). Sin embargo, se conoce que existen especies que regulan su metabolismo en función de la escasez de oxígeno (Hill, 1980), como es el caso del Guppy lebistes reticulatus, el cual es capaz de sobrevivir a concentraciones muy bajas de oxígeno disuelto (Merino y Droste, 1983). Por otro lado, Boyd (1990) menciona que el carbono que es transformado en materia orgánica producto del proceso de fotosíntesis es equivalente a la concentración de oxígeno disuelto presente en el agua.

\section{Nitratos}

Los resultados indican que los valores de nitratos sobrepasaron el valor establecido de $13 \mathrm{mg} / \mathrm{L}$ en la categoría 4 del ECA para agua. Esto podría deberse a las excretas de los caballos que se encuentran en la zona y a la intensa actividad biológica de fauna y flora en esta laguna. El nitrato forma parte del ciclo natural del nitrógeno, pero su presencia en los suelos y su solubilidad en el agua hace que alcance grandes concentraciones

Tabla 6. Resultados de los parámetros fisicoquímicos y microbiológicos obtenidos en la laguna Marvilla, Pantanos de Villa, en octubre de 2020.

\begin{tabular}{|c|c|c|c|c|c|c|c|c|c|c|}
\hline & pH & $\begin{array}{l}\text { C. E. } \\
(\mu \mathrm{SI} \\
\mathrm{cm})\end{array}$ & $\begin{array}{c}\text { TDS } \\
\text { (mg/L) }\end{array}$ & $\mathrm{T}\left({ }^{\circ} \mathrm{C}\right)$ & $\begin{array}{l}\text { O.D } \\
(\mathrm{mg} / \mathrm{L})\end{array}$ & $\begin{array}{l}\text { Nitratos } \\
\text { (mg/L) }\end{array}$ & $\begin{array}{c}\text { Amoniaco } \\
\text { total } \\
\text { (mg/L) }\end{array}$ & $\begin{array}{c}\text { Fósforo } \\
\text { total } \\
\text { (mg/L) }\end{array}$ & $\begin{array}{l}\text { DBO5 } \\
\text { (mg/L) }\end{array}$ & $\begin{array}{c}\text { Coliformes } \\
\text { termotolerantes } \\
\text { (NMP/100 mL) }\end{array}$ \\
\hline PM-01 & 9,1 & 4600,0 & 3381,0 & 26,2 & 18,1 & 150,6 & 0,0 & 0,2 & 22,9 & 3300,0 \\
\hline PM-02 & 8,5 & 4520,0 & 3322,2 & 26,1 & 10,3 & 97,5 & 5,1 & 0,2 & 14,3 & 33000,0 \\
\hline PM-03 & 8,7 & 4730,0 & 2476,6 & 24,1 & 11,8 & 150,6 & 0,0 & 4,0 & 13,1 & 17000,0 \\
\hline $\begin{array}{l}\text { Promedio y desviación } \\
\text { estándar }\end{array}$ & $\begin{array}{c}8,8 \pm \\
0,3\end{array}$ & $\begin{array}{c}4616,7 \pm \\
106,0\end{array}$ & $\begin{array}{c}3059,9 \pm \\
506,0\end{array}$ & $\begin{array}{c}25,5 \pm \\
1,2\end{array}$ & $13,4 \pm 4,1$ & $132,9 \pm 30,7$ & $1,7 \pm 3,0$ & $1,5 \pm 2,2$ & $16,8 \pm 5,3$ & $\begin{array}{c}17766,7 \pm 14 \\
864,8\end{array}$ \\
\hline
\end{tabular}


en ríos o lechos profundos; su incremento puede estar influenciado por las actividades humanas (Bolaños et al., 2017), ya sea por presencia de fertilizantes o aguas residuales domesticas o industriales (Larios, 2009). En consecuencia, las altas concentraciones de nitrógeno orgánico y amoniacal pueden generar un proceso de eutrofización. Al oxidarse, estos compuestos producen nitritos y nitratos que consumen rápidamente el oxígeno disuelto, en un proceso que depende de la temperatura, el oxígeno disuelto y el pH (Carranza, 2001). Sin embargo, las altas concentraciones de nitritos y nitratos resultan tóxicas para la biota presente, y el consumo desmesurado de oxígeno en el agua es perjudicial para la supervivencia de las comunidades acuáticas (Roldán, 2008).

\section{Amoniaco total}

La concentración de amoniaco total en la laguna Marvilla solo se presentó en el PM-02 con 5,11 mg/L. Este valor sobrepasa el límite establecido en la categoría 4 del ECA para agua de 0,12 mg/L que se halla en función del $\mathrm{pH}$ y la temperatura. Sin embargo, la ausencia de amoniaco total en los otros puntos de muestreo se debe probablemente a la dilución de la carga que llega del canal sur hasta los extremos donde se ubican el PM-1 y el PM-3. El nitrógeno amoniacal es un contaminante que provoca diversos efectos en el ambiente (API, 1981). No obstante, su presencia es común en el ciclo del nitrógeno y se puede encontrar en el suelo, el agua y el aire. Debido a su reactividad, esta sustancia no dura mucho en su forma pura (ATSDR, 2004). Las fuentes de amoniaco pueden ser naturales como el crecimiento de algas, el decaimiento de la materia de la planta o animal, los desechos animales y la fijación de nitrógeno; y antropogénicas, como vertidos de aguas residuales domésticas, industriales y de origen agrícolas (EPA, 2013). Sin embargo, su detección en cantidad significativa se considera como indicio de probable contaminación reciente (Fernández y Vásquez, 2006).

\section{Demanda bioquímica de oxígeno}

Los resultados señalan que los valores de demanda bioquímica de oxígeno sobrepasaron el límite establecido de $5 \mathrm{mg} / \mathrm{L}$ en la categoría 4 del ECA para agua. En el estudio realizado por Lecca y Lizama (2014) se menciona que una concentración alta de materia orgánica favorece el crecimiento de hongos y bacterias. El oxígeno es requerido por la materia orgánica para ser utilizado en su oxidación, por lo que al ser consumido altera el correcto desarrollo de la fauna y la flora acuáticas que necesitan de él. Además, se han identificado otros efectos al ecosistema acuático, y se encontró un cambio en la calidad del agua, un posible aumento en el potencial de hidrogeno $(\mathrm{pH})$, que causa una merma en la vida de flora y fauna.

\section{Fósforo total}

De acuerdo con los resultados, los valores de fósforo total sobrepasaron el límite establecido de 0,035 mg/L en la categoría 4 del ECA para agua. Jiménez (2000) refiere que un aumento de fósforo total en un cuerpo de agua está relacionado con el uso de abonos fosfatados y detergentes. Además, menciona que un aumento repentino de este nutriente puede llevar a un crecimiento descontrolado de biomasa acuática, un proceso conocido como eutrofización. En la eutrofización aumenta la cantidad de plantas acuáticas, disminuye el oxígeno disuelto y cambia el contenido de $\mathrm{pH}$, lo que causa una afectación en la calidad del agua (EPA, 2000).

\section{Coliformes termotolerantes}

Los resultados indican que los valores de coliformes termotolerantes sobrepasaron el límite establecido de 1000 mg/L en la categoría 4 del ECA para agua. La cantidad de coliformes termotolerantes en el agua nos permite identificar la contaminación patogénica y bacteriológica del agua proveniente de residuos fecales (Pérez et al., 2017). La cercanía del humedal con urbanizaciones, fábricas y camales probablemente genera su contaminación por aguas residuales que llegan a través de los canales, al igual que su contaminación por excretas provenientes de los caballos de los alrededores de la laguna y de la avifauna que habita en el área. Esta problemática se asemeja con el estudio realizado por Rodríguez et al. (2017) en el humedal de Ventanilla, donde se evidencia que la interacción del humedal con la población genera contaminación del agua, caracterizada por poseer una rica fuente de nutrientes que permite el desarrollo de comunidades bacterianas, las que, a su vez, aseguran la sobrevivencia de los coliformes termotolerantes; además, mencionan que la contaminación por coliformes termotolerantes, en un futuro, podrían ocasionar cambios en la composición, estructura y dinámica del ecosistema.

\section{Índice de calidad del agua}

Mediante el cálculo de la fórmula del ICA-PE se determinó que la calidad de la laguna Marvilla es regular, al obtener un valor de 46,3 (tabla 7). Al interpretar la clasificación del ICA-PE, la ANA menciona que un agua con calidad regular se genera por ser ocasionalmente amenazada o dañada y su calidad no presenta los valores deseables. Sin embargo, este resultado no es definitivo para señalar el estado actual de la laguna Marvilla, puesto que, de los 17 parámetros requeridos en la categoría 4, se utilizaron solo seis. Por otro lado, los valores límites de los

Tabla 7. Índice de calidad de agua de la laguna Marvilla.

\begin{tabular}{cc}
\hline Factores & Valor \\
\hline Alcance (F1) & 0,7 \\
\hline Frecuencia (F2) & 0,7 \\
\hline Amplitud (F3) & 92,9 \\
\hline ICA & 46,3 \\
\hline Fuente: ANA (2018).
\end{tabular}


parámetros evaluados en la categoría 4 del ECA para agua establecen valores para lagos y lagunas de agua dulce, no para lagunas salobres como la laguna Marvilla. Por ello, algunos de los parámetros solicitados en esta categoría no cumplen con la normativa.

\section{Conclusiones}

La laguna Marvilla presentó un agua salobre, que tuvo una condición neutra en los meses donde el nivel de agua fue alto, y ligeramente alcalina cuando el nivel de agua fue bajo. La mayoría de los parámetros evaluados sobrepasan lo establecido en la categoría 4 del ECA para agua, debido posiblemente a las condiciones en la que se sitúa la laguna Marvilla. Por ello, las descargas de aguas residuales que desembocan en la laguna, la presencia de excretas de los animales que se encuentran a sus alrededores y el arrojo de residuos sólidos podrían estar ocasionando que estos parámetros sean alterados. Sin embargo, la laguna presentó niveles óptimos de oxígeno disuelto, los cuales permiten un mayor desarrollo de la vida acuática. Por otro lado, la laguna Marvilla presenta condiciones naturales que generan que parámetros como la conductividad eléctrica siempre sean altos $y$, por ende, sobrepasen el valor permisible. Finalmente, el cálculo del índice de calidad de agua nos permitió determinar que el agua de la laguna Marvilla presenta una calidad regular, la cual se ve influenciada por los parámetros de $\mathrm{DBO}_{5}$, fósforo total, amoniaco total y coliformes termotolerantes, que excedieron su valor permisible.

\section{Recomendaciones}

Se recomienda que el ECA para Agua aprobado por el Decreto Supremo 004-2017-MINAM incorpore una nueva subcategoría para lagunas salobres, ya que en dicha normativa se consideran valores generales para la categoría 4 («Conservación del ambiente acuático»), subcategoría E1: «Lagos y lagunas». Esto traerá como resultado que los parámetros comparados no cumplan de la misma forma para lagunas con características particulares, como es el caso de la laguna Marvilla, que se ubica cerca del mar y presenta valores de conductividad eléctrica que sobrepasan lo establecido por el ECA.

Se debe empezar a generar líneas base que aporten a investigaciones futuras, puesto que esta data almacenada contribuiría a mejorar la gestión del humedal, tomando en cuenta los posibles riesgos de contaminación por presencia de un aumento inesperado de determinados parámetros en la calidad del agua.

\section{Agradecimientos}

El presente artículo se realizó gracias al apoyo de especialistas del área ambiental de Prohvilla y de Dámaso Ramírez, profesor de la Universidad Científica del Sur.

\section{Fuente de financiamiento}

Este trabajo tuvo el soporte logístico de Prohvilla.

\section{Contribución de autoría}

SH-V, ML-E y MP-V contribuyeron en el procesamiento de la información, el análisis de los resultados y la redacción del artículo. DCh-A contribuyó en la gestión de la información, el procesamiento de la información, el análisis de los resultados y la redacción del artículo. Todos los autores aprobaron la versión final del texto.

\section{Conflictos de interés}

Los autores declaran que no existe ningún conflicto de interés.

\section{Referencias bibliográficas}

Agency for Toxic Substances and Disease Registry (ATSDR). (2004) Public Health Statement for Ammonia. Disponible en: https://www. atsdr.cdc.gov/ToxProfiles/tp126-c1-b.pdf

Álvarez, C. (2016). Determinación analítica de detergentes en las aguas de los Pantanos de Villa (tesis de Ingeniería Química). Pontificia Universidad Católica del Perú, Lima. Disponible en: http://tesis. pucp.edu.pe/repositorio/handle/20.500.12404/7570

API. (1981). The sources, chemistry, fate and effects of ammonia in aquatic environments. Washington, D. C.: American Petroleum Institute.

Arana, C. y Salinas, L. (2003). «Flora vascular de los humedales de Chimbote, Perú». Revista Peruana de Biología, 10(2), pp. 221-224. Disponible en: http://www.scielo.org.pe/scielo.php?script=sci_ arttext\&pid=S1727-99332003000200014

Autoridad Nacional del Agua (ANA). (2016). Protocolo nacional para el monitoreo de la calidad de los recursos hídricos superficiales. Lima: Autoridad Nacional del Agua. Disponible en: https://www.ana.gob. pe/publicaciones/protocolo-nacional-para-el-monitoreo-de-lacalidad-de-los-recursos-hidricos-0

Autoridad Nacional del Agua (ANA). (2018). Metodología para la determinación del índice de calidad de agua ICA-PE aplicado a los cuerpos de agua continentales superficiales. Lima: Autoridad Nacional del Agua. Disponible en: https://www.ana.gob.pe/publicaciones/ metodologia-para-la-determinacion-del-indice-de-calidad-deagua-ica-pe-aplicado-los

Bolaños, J., Cordero, G. y Segura, G. (2017). Determinación de nitritos, nitratos, sulfatos y fosfatos en agua potable como indicadores de contaminación ocasionada por el hombre, en dos cantones de Alajuela (Costa Rica). Tecnología en Marcha, 30(4), pp. 15-27. Disponible en: http://dx.doi.org/10.18845/tm.v30i4.3408

Bopp, G. y Peláez, F. (2019). «Evaluación de la flora vascular de los humedales costeros de La Libertad, Perú». Revista de Investigación Científica de la Universidad Nacional de Tumbes, 16(2), pp. 151-156. DOI: 10.17268/manglar.2019.021.

Boyd, C. (1990). Water quality in ponds for aquaculture. Alabama: Birmingham Publishing.

Carranza, R. (2001). Medio ambiente: problemas y soluciones. Lima: Universidad Nacional del Callao. 
Castillo, R. y Huamantinco, A. (2020). «Variación espacial de la comunidad de macroinvertebrados acuáticos en la zona litoral del humedal costero Santa Rosa, Lima, Perú». Revista Biología Tropical, 68(1), pp. 50-68. DOI: 10.15517/RBT.V68I1.35233.

CCME. (2001). Canadian water quality guidelines for the protection of aquatic life: CCME Water Quality Index 1.0. Technical Report. Disponible en: http://ceqg-rcqe.ccme.ca/download/en/137

Comisión Nacional del Agua. (2000). Ley Federal de Derechos. Disposiciones aplicables en materia de aguas nacionales 2019. Ciudad de México: Secretaría de Medio Ambiente y Recursos Naturales. Disponible en https://files.conagua.gob.mx/conagua/ publicaciones/Publicaciones/CGRF-1-19\%20LFD.pdf

Cordy, G. (2001). «A primer on water quality». Disponible en: https:// pubs.usgs.gov/fs/fs-027-01/pdf/FS-027-01.pdf

Cruz, F., Burger, H. y Borgesa, R. (2007). «Evaluación de aves en la laguna El Paraíso, Lima, Perú». Revista Peruana de Biología, 14(1), pp. 139-144. Disponible en: https://www.researchgate.net/ publication/242240700_Evaluacion_de_aves_en_la_laguna_El_ Paraiso Lima Peru

Environmental Protection Agency (EPA). (2000). National Water Quality Inventory. 2000 Report. Washington, D. C.: United States Environment Protection Agency. Disponible en: https://www.epa. gov/sites/production/files/2015-09/documents/2000_national_ water_quality_inventory_report_to_congress.pdf

Environmental Protection Agency (EPA). (2013). Aquatic life ambient water quality criteria for ammonia - Freshwater. 2013. Washington, D. C.: United States Environment Protection Agency. Disponible en: https://www.epa.gov/sites/production/files/2015-08/documents/ aquatic-life-ambient-water-quality-criteria-for-ammoniafreshwater-2013.pdf

Sistema Nacional de Información Ambiental. (2017). «Decreto Supremo N. ${ }^{\circ}$ 004-2017-MINAM. Aprueban Estándares de Calidad Ambiental (ECA) para agua y establecen disposiciones complementarias». Disponible en: https://sinia.minam.gob. pe/normas/aprueban-estandares-calidad-ambiental-eca-aguaestablecen-disposiciones

Fajardo, N. (2018). Evaluación de la calidad microbiológica y fisicoquímica de las aguas en el Área de Conservación Regional Humedales de Ventanilla, región Callao, Perú (tesis de maestría en Ciencias Ambientales). Universidad Nacional Mayor de San Marcos, Lima. Disponible en: https://cybertesis.unmsm.edu.pe/ handle/20.500.12672/7738

Fernández. M. y Vásquez, Y. (2006). «Origen de los nitratos $\left(\mathrm{NO}_{3}\right)$ y nitritos $\left(\mathrm{NO}_{2}\right)$ y su influencia en la potabilidad de las aguas subterráneas». Minería y Geología, 22(3). Disponible en: http:// revista.ismm.edu.cu/index.php/revistamg/article/view/122/0

Finlayson, C., D'Cruz, R. y Davidson. N. C. (2005). Ecosystems and human well-being: Wetlands and water wynthesis. Washington, D. C.: World Resources Institute. Disponible en: https://www. millenniumassessment.org/documents/document.358.aspx.pdf

Jiménez, A. A. (2000). «Determinación de los parámetros físicoquímicos de calidad de las aguas». Revista Interdisciplinar de Cestión Ambiental, 2(23), pp. 12-19. Disponible en: http://ocw.uc3m.es/ ingenieria-quimica/ingenieria-ambiental/otros-recursos-1/OR-F001.pdf

Groel, N. (2006). «Conductividad». Sociedad Acuicultora de la Plata. Disponible en: http://www.sadelplata.org/articulos/groel_060910. pdf

Hill, R. W. (1980). Fisiología animal comparada. Barcelona: Reverté. Disponible en: https://books.google.es/ books? hl=es\&lr=\&id=w7aoEY- $48 E C \& o i=f n d \& p g=P A 1 \& d q=h i l l+$ fis iolog\%C3\%ADa+animal+comparada\&ots=sbcOfhcSXJ
Instituto Geológico, Minero y Metalúrgico (Ingemmet). (2019). Estudio hidrogeológico del Refugio de Vida Silvestre Los Pantanos de Villa (RVSPV). Lima: Ingemmet. Disponible en: https://repositorio. ingemmet.gob.pe/handle/20.500.12544/2241?locale=es

Larios, L. (2009). «Contaminación del agua por nitratos: significación sanitaria». Revista Archivo Medico de Camagüey, 13(2). Disponible en: http://scielo.sld.cu/scielo.php?script=sci arttext\&pid=S1025-02552009000200017

Lecca, E. y Lizama, E. (2014). «Caracterización de las aguas residuales y la demanda bioquímica de oxígeno». Industrial Data, 17(1), pp. 71-80. Disponible en: https://www.redalyc.org/pdf/816/81640855010.pdf

Merino, F. y Droste, H. J. (1983). «Adaptación del Guppy Lebistes reticulatus al ambiente II. La adaptación fisiológico-metabólica». Actualidades Biológicas, 12(45), pp. 68-76. Disponible en: https:// revistas.udea.edu.co/index.php/actbio/article/view/330312

Paredes, D. (2010). Determinación de amenazas en humedales urbanos: Estudio de tres humedales de Valdivia, Chile (tesis en Ingeniería en Conservación de Recursos Naturales). Universidad Austral de Chile, Valdivia. Disponible en: http://cybertesis.uach.cl/tesis/uach/2010/ fifp227d/doc/fifp227d.pdf

Pérez, B. A. y Álvarez, M. Ñ. (2018). «Índice de calidad del agua según NSF del humedal laguna Los Milagros (Tingo María, Perú)». INDES Revista de Investigación para el Desarrollo Sustentable, 2(2), pp. 98107. Disponible en: http://revistas.untrm.edu.pe/index.php/INDES/ article/view/81/194

Pérez, R., Riveiro, F., Jiménez, M., Manganiello, L., Vega, C., Covad, R. y Moreno, J. (2017). «Water quality assessment in a Caribbean saltwater wetland». Revista Ingeniería UC, 24(3), pp. 417-427.

Pulido, V. y Bermúdez, L. (2018). «Estado actual de la conservación de los hábitats de los Pantanos de Villa, Lima, Perú». Arnaldoa, 25(2), pp. 679-702. Disponible en: http://www.scielo.org.pe/pdf/arnal/ v25n2/a19v25n2.pdf

Rodríguez, R., Retamozo, R., Aponte, H. y Valdivia, E. (2017). «Evaluación microbiológica de un cuerpo de agua del ACR Humedales de Ventanilla (Callao, Perú) y su importancia para la salud pública local». Ecología Aplicada, 16(1), pp. 15-21.

Roldán, P. (2008). «Fundamentos de limnología neotropical». Antioquia: Universidad de Antioquia.

Russi, D., Brink, P. T., Badura, T. et al. (2013). «The economics of ecosystems and biodiversity for water and wetlands». Londres y Bruselas: IEEP. Disponible en: https://www.ramsar.org/sites/ default/files/documents/pdf/TEEB/TEEB Water-Wetlands FinalConsultation-Draft.pdf

Sajurjo, E. (2001). Valoración económica de servicios ambientales prestados por ecosistemas: Humedales en México. Ciudad de México: Intituto Nacional de Ecología. Disponible en: http://www2.inecc. gob.mx/dgipea/descargas/pea-ri-2001-001.pdf

Servicio Nacional de Áreas Naturales Protegidas por el Estado (Sernanp). (2013). «Humedales en Áreas Naturales Protegidas, fuentes de vida y desarrollo». Disponible en: https://www.sernanp. gob.pe/documents/10181/104923/0HUMEDALES+\%284\%29.pdf/ fa45aa19-1670-401f-91f6-ac667eaaf513

Valdivieso, A. (2018). «¿Qué es agua salobre?». laguas. Disponible en: https://www.iagua.es/respuestas/que-es-agua-salobre

Water Quality Association. (2010). «Glossary of salt water: Water classifications». Disponible en: http://www.wqa.org/

Water Reuse. (2007). «Salinidad y calidad de agua». Disponible en: $\quad$ http://www.salinitymanagement.org/Salinity\%20 Management\%20Guide/ls/ls 3d.html 